\title{
Use of Recombinant Factor VII in Cardiac Surgery
}

Mark Elizalde PharmD, Leonid Slobodskoy* PharmD, Michael Diodato MD, James Chang $M D$ and Edgar G. Chedrawy* MD

*Please send correspondence to:

Edgar G. Chedrawy MD, MSc, FRCSC, FACS

Associate Professor of Surgery, University of Illinois at Chicago and

Medical Director, Cardiovascular Thoracic Surgery, Weiss Memorial Hospital

4646 N Marine Dr.,

Chicago, IL

60640

Phone: 773-564-5912

Fax: 312-996-2013 


\begin{abstract}
The off-label use of recombinant human coagulation factor VIIa has been increasing to a number of different treatment areas since its original approval in 1999. Several US patents describe claims for FVIIa utilization in non-hemophilia patients, treatment of bleeding due to trauma, as a means to reverse major bleeding, including intracranial bleeding, induced from fibrinolytic therapy as well as a patent for using FVIIa in the treatment of bleeding for patients with bleeding disorders not caused by hemophilia, but rather bleeding disorders due to thrombocytopenia, platelet disorders, and von Willebrand's disease. Bleeding after cardiac surgery remains a serious complication that can increase both morbidity and mortality. We review the off-label usage of recombinant factor VIIa as a hemostatic agent that may help control bleeding following cardiac surgery.
\end{abstract}

Keywords: Cardiac Surgery, Factor VIIa, Hemostasis, Off-label

Short Running Title: Recombinant Factor VII and Cardiac Surgery 


\section{Introduction}

Recombinant factor VIIa (rFVIIa) NovoSeven; NovoNordisk) was originally approved by the US Food and Drug Administration (FDA) in 1999 for treating bleeding episodes in hemophilia A or B patients with inhibitors (antibodies) to Factor VIII or Factor IX. In 2005 the US FDA extended the indication to include treatment and prevention of bleeding episodes due to surgical procedures in patients with acquired hemophilia or congenital factor VII deficiency [1]. Medical literature has described a number of clinical experiences using rFVIIa in non-hemophilia coagulopathies such as in surgery, trauma, intracerebral hemorrhage, liver disease, and gastrointestinal bleeding [2,3]. There are also several US patents describing claims for FVIIa utilization in non-hemophilia patients $[4,5,6]$. Axelsen et al. describes using FVIIa or a FVIIa equivalent for the manufacture of a medication treatment of bleeding due to trauma [4]. Another patent by Skamira et al. suggests FVIIa may be used as a means to reverse major bleeding, including intracranial bleeding, induced from fibrinolytic therapy [5]. A third patent by Hedner, describes using FVIIa in the treatment of bleeding for patients with bleeding disorders not caused by hemophilia, but rather bleeding disorders due to thrombocytopenia, platelet disorders, and von Willebrand's disease [6]. The purpose of this article is to focus specifically on the off-label use of rFVIIa in cardiac surgery.

\section{Pharmacology and Mechanism of Action of Factor VII}

Activated factor VIIa (FVIIa) is a naturally occurring substance responsible for initiating local hemostasis by complexing with tissue factor (TF). The FVIIa-TF complex activates factor X and factor IX [2]. Activated factor $\mathrm{X}$ (factor $\mathrm{Xa}$ ) complexes with factor $\mathrm{V}$, calcium, and phospholipids to convert prothrombin to thrombin. Thrombin is responsible for converting fibrinogen to fibrin which forms a thrombus with platelets at the site of injury [1]. rFVIIa is structurally almost identical to natural plasma FVIIa (nFVIIa) and is thought to function in the same way. Recombinant FVIIa displays linear pharmacokinetics and its half life is approximately 2 hours [2,3]. The activity of rFVIIa may be affected by temperature, $\mathrm{pH}$ and calcium levels. Calcium plays an important role in rFVIIa activity, but correlating the effect with serum levels isn't yet well established. Hypothermia (temperature decrease from $37-33^{\circ} \mathrm{C}$ ) has been shown to decrease rFVIIa-TF complex activity by about $20 \%$ and acidosis ( $\mathrm{pH}$ decrease from 7.4 to 7.0) has been shown to decrease rFVIIa activity by about 90\% [2]. When rFVIIa is given at pharmacological doses it is thought to exceed nFVIIa levels by greater than 100 times and may exert pharmacological effects that are not observed with nFVIIa at normal physiological levels. Proposed mechanisms for these effects include:

- $\quad$ TF-dependent mechanism: rFVIIa complexes with TF to activate factor $\mathrm{X}$ leading to thrombin production at the site of injury. 
- $\quad$ TF-independent mechanism: rFVIIa binds to activated platelets to activate factor X leading to thrombin production. This mechanism provides a localized initiation of hemostasis as well, because only activated platelets allow for binding. This is the mechanism most likely responsible for "bypassing" the need for FVIII or FIX in hemophiliac patients.

- $\quad$ Plasma FVII may compete with FVIIa for binding to TF and pharmacological doses of rFVIIa may overcome this inhibitory effect of plasma FVII on coagulation.

- Recombinant FVIIa may down regulate the fibrinolytic system through enhanced activation of thrombin activatable fibrinolysis inhibitor [2].

\section{Cardiac Surgery and Factor VII}

The use of rFVIIa in cardiac surgery is somewhat controversial. Cardiac disease itself and the process of using cardiopulmonary bypass (CPB) in cardiac surgery can increase the risk of myocardial ischemia and thrombosis. TF has been found on atherosclerotic plaques as well as activated monocytes in the blood of cardiac surgery patients [3]. There is evidence to suggest that increased thrombin generation observed during $\mathrm{CPB}$, despite high unfractionated heparin (UFH) and tissue factor pathway inhibitor (TFPI) levels, may be caused by FVIIa complexing with TF [7]. Increased thrombin generation can persist even after CPB [3]. Interestingly, antagonism of FVIIa has been used experimentally in animal models as a potential new target for preventing thrombosis from atherosclerotic plaques [8]. On the other hand, bleeding after cardiac surgery remains a serious complication. CPB has been shown to decrease the number and function of platelets with an average decline of about $50 \%$. Levels of coagulation factors (II, V, VII, IX, X, XIII) may fall during CPB as well, which is likely due to hemodilution. Enhanced fibrinolysis may occur in some patients as a result of CPB and there is also potential of heparin rebound, due to protamine having a shorter half-life than heparin, which could attribute to bleeding complications [9]. Despite potential risks for thrombosis, bleeding complications from cardiac surgery still occur and rFVIIa has been used off-label as treatment [9-13].

In a recent review Despotis et al. from Washington University in St. Louis discussed the management of post cardiac surgery bleeding [14]. The normal hemostatic mechanisms are purposefully altered during the operation and return to homeostasis can be delayed. Reexploration due to microvascular bleeding has been associated with a three-fold increase in mortality and multiple transfusions have been associated with end organ injury [15,16]. The authors state that post-operative bleeding is multifactorial including, hereditary, iatrogenic, and related to preexisting medical conditions or acquired during the surgical intervention. Furthermore, the risk of postoperative bleeding is enhanced by procedure type, duration of bypass and additional patient related factors [17]. 
Management of postoperative bleeding has traditionally involved the use of blood products. Guidelines for the use of FFP, cryoprecipitate and platelets have been published by both the NIH and American Association of Anesthesiologists [18,19]. Hypothermia and severe acidosis should be corrected prior to institution of routine hemostatic therapy. Factor concentrates and pharmacologic agents such as DDAVP and conjugated estrogens have also been employed in the management of postoperative bleeding. Recombinant activated Factor VIIa (rFVIIa) currently has been approved by the FDA for the management of bleeding in hemophilia A and B patients. In an ex vivo experiment, Sorenson et al. confirmed that by adding a combination of rFVIIa and fibrinogen to 18 post cardiopulmonary bypass (CPB) samples all thromboelastometric parameters were corrected to the preoperative range [20]. Currently, there are no guidelines for the use of factor concentrates in the setting of uncontrolled bleeding and decision are left to the physician on a patient by patient basis. The off-label use of these agents has been reported to be successful in multiple clinical situations. As of the writing of their article, the authors stated that few adequately controlled studies existed for the complications following use of rFVIIa in cardiac surgical patients and that the findings of previous studies needed to be critically reviewed.

Safety, efficacy and dosing have yet to be established for this off-label role of rFVIIa. Gill et al. attempted to study two different doses of rFVIIa in cardiac surgery patients requiring CPB where conventional transfusion therapy was indicated. One hundred seventy nine patients were randomized to receive placebo, $40 \mathrm{mcg} / \mathrm{kg}$ or $80 \mathrm{mcg} / \mathrm{kg}$ of rFVIIa. Fewer patients receiving rFVIIa underwent reoperations for bleeding (placebo, 25\%; 40mcg/kg rFVIIa, $14 \%$ [P = 0.21]; $80 \mathrm{mcg} / \mathrm{kg}$ rFVIIa, $12 \%$ [P = 0.04]). Also, the use of rFVIIa was associated with fewer allogeneic blood transfusion volumes (placebo, $825 \mathrm{~mL}$ [25\% to 75\% interquartile range (IQR), 326.5 to $1893 \mathrm{~mL}$ ]; $40 \mathrm{mcg} / \mathrm{kg}$ rFVIIa, $640 \mathrm{~mL}$ [25\% to $75 \%$ IQR, 0 to $1920 \mathrm{~mL}$ ], P $=0.047 ; 80 \mathrm{mcg} / \mathrm{kg}$ rFVIIa, $500 \mathrm{~mL}$ [ $25 \%$ to $75 \%$ IQR, 0 to $1750 \mathrm{~mL}$ ], $\mathrm{P}=0.042$ ). More cases of adverse events (e.g. death, cerebral infarction, myocardial infarction and pulmonary embolism) occurred in patients that received rFVIIa, but there were not enough total adverse events to conduct statistical analysis. There were 18 total adverse events and 14 deaths (placebo, 4 deaths [6\%]; combined rFVIIa dose groups, 10 deaths [10\%]). Unfortunately the results of this study were not statistically significant, because the study was underpowered [10].

Massud et al reported a study of 93 who received rFVIIa after cardiovascular surgery for the management of refractory bleeding [21]. Patients received an average of $7.6 \pm 6.8$ units of red blood cells (RBCs) before rFVIIa intervention. Blood product consumption was significantly reduced in the six hours after dosing and no adverse thrombotic events were recorded. The authors concluded that although effective in their study, optimal dosing possible adverse events needed further investigation. Willis et al. performed an Australian multi-institutional reviewing 804 cases of rFVIIa use with multiple dosing points. They concluded that no significant differences existed in the rate of thromboembolic adverse events, response to bleeding or 28-day 
mortality amongst different dosing cohorts and that smaller doses may be as effective as larger doses [22].

In 2011, Chapman AJ, et al retrospectively analyzed the safety profile and efficacy of rFVIIa used for bleeding following cardiac surgery [23]. 236 patients receiving rFVIIa were compared with 213 patients who undergone similar cardiac procedures and did not receive rFVIIa. Primary endpoints were thromboembolic events, mortality, incidence of reoperation, use of blood products, and patient disposition at 30days. Results showed no statistical difference in the incidence in thrombi-embolic events, or acute renal failure in the two groups. Mortality rates were also not significantly different ( $\mathrm{rFVIIa} 7.7 \%$ and control group $4.3 \%$, $\mathrm{p}=0.14$ ). The rFVIIa group had a higher rate of re-operation for bleeding ( $11 \%$ vs. $4 \%)$ and also required increased traditional blood product administration. They concluded that rFVIIa is an effective hemostatic agent with an acceptable safety profile as it does not appear to be associated with increased postoperative complications, such as thromboembolic events and death.

In contrast, Zangrillo et al have published 2 meta-analyses regarding the use of rFVIIa in the post cardiac surgical patient. In 2009, the authors performed a meta-analysis of 5 trials that included 298 patients. The authors observed a statistically insignificant reduction in the rate of surgical reexploration (13\%) in the rFVIIa group vs. (57\%) in the control group, with a trend toward an increase in the rate of perioperative stroke ( $5 \%$ vs. $1.4 \%$,respectively) and no effect on mortality (15\% in both groups) [24]. In 2011, this same group reviewed 470 patients (254 receiving rFVIIa and 216 controls) [25]. The use of rFVIIa was associated with an increased rate of stroke (4.7\%) in the rFVIIa group vs. $0.9 \%$ in the control, with an insignificant reduction in rate of surgical reexploration ( $13 \%$ vs. $42 \%, \mathrm{p}=0.19)$. The authors observed a trend toward an increase in perioperative thromboembolic events ( $7.5 \%$ in the rFVIIa group vs. $5.6 \%), p=0.14$ ). No difference in mortality was observed between groups. They concluded that great care must be exercised with the use of rFVIIa and further studies needed to be performed to identify its' safety and efficacy in the post CPB patient.

Romagnoli et al. studied the use of small dose (1.2mg) of rFVIIa in 15 patients with intractable bleeding following cardiac surgery and compared them to 15 similar patients retrospectively, from before their institution started using rFVIIa in cardiac surgery. The primary endpoint was the amount of blood loss, which was described as hourly bleeding or as 1-3-5-24 hour bleeding. Secondary endpoints were considered following the study's standard transfusion protocol, and included the need for additional transfusions of red blood cells, fresh frozen plasma, and platelets. The patients who received rFVIIa had significantly less postoperative bleeding with median 25th-75th 24-hour blood loss percentiles of 1685 (1590-1770) $\mathrm{mL}$ for the study group versus $3170(2700-3850) \mathrm{mL}$ for the control group $(\mathrm{P}=0.0004)$. Patients that received rFVIIa also had reduced blood product transfusion requirements when compared to the control patients; red blood cells (7 [4-8] U versus18 [12-21] U; P = 0.001), fresh frozen plasma (7.5 [6 -11] U versus $11[9-15] \mathrm{U} ; \mathrm{P}=0.003)$ and platelets $(0[0-4] \mathrm{U}$ versus 9 [6 -13] $\mathrm{U} ; \mathrm{P}=0.001)$. Three 
patients died in the control group while none died in the study group; however, this was not statistically significant. There were significantly more complications in the study group (33.3\%, $\mathrm{n}=5$ versus $20 \%, \mathrm{n}=3$ ). Complications included: stroke ( 2 in study group), renal failure ( 1 in study group and 1 control), respiratory failure (1 in study group), low-output syndrome (1 in study group and 1 control), and infection (1 in study group) [13].

Tatoulis et al. studied the safety of rFVIIa using the dose of $100 \mathrm{mcg} / \mathrm{kg}$ as a "rescue" therapy in patients undergoing cardiac surgery when bleeding could not be controlled despite conventional blood products and medications. Of the 30 patients that received rFVIIa, none of which developed any thrombotic events. Patients showed a reduction in the requirement of additional blood products; red blood cells (5.4 [0-15] U versus 0.9 [0 -4] U; P < 0.001), cryoprecipitate (10.5 [0-32] U versus 1.5 [0 - 10] U; P < 0.001), platelets (12.7[0-40] U versus 0.5 [0 -5] U; P < 0.001 ), and fresh frozen plasma (9.7 [5-20] U versus 1.1 [0-14] U; $\mathrm{P}<0.001$ ) pre-rFVIIa versus post-rFVIIa, respectively. Ten patients didn't require any additional red blood cell products after rFVIIa was administered. Recombinant FVIIa significantly affected the coagulation profile by reducing INR (1.5 [1.1-8] versus 0.95 [0.7-2.6]; P < 0.001) and APTT (52 [26-220] versus 39 [22-104]; P = 0.008) pre-rFVIIa versus post-rFVIIa respectively [12].

In a Canadian review by Karkouti et al., the off-label use of rFVIIa in cardiac surgery was retrospectively analyzed in 503 patients from multiple centers. In this review, rFVIIa was used primarily to control bleeding when standard measures had failed. The doses were not uniform and appeared to be directly related to the severity of bleeding, but the median total dose was $62 \mathrm{mcg} / \mathrm{kg}$ (quartile 1 [Q1], quartile 3 [Q3], $40,89 \mathrm{mcg} / \mathrm{kg}$ ). The use of $\mathrm{rFVIIa}$ was associated with a reduction in blood product transfusion rates; red blood cells $(8[5,12] \mathrm{U}$ versus $2[1,5] \mathrm{U}$; $\mathrm{P}<0.0001)$, cryoprecipitate $(0[0,10] \mathrm{U}$ versus $0[0,10] \mathrm{U} ; \mathrm{P}<0.0001)$, platelets $(10[10,15] \mathrm{U}$ versus $5[0,10] \mathrm{U} ; \mathrm{P}<0.0001)$, and fresh frozen plasma $(8[5,12] \mathrm{U}$ versus $2[0,6] \mathrm{U} ; \mathrm{P}<0.0001)$ pre-rFVIIa versus post-rFVIIa, respectively. The authors mentioned that the effectiveness of rFVIIa may be increased when it is given early in the course of refractory bleeding. The adverse events were as follows: mortality, 32\% $(n=159)$; stroke, $12 \%(n=58)$; renal failure, $12 \%(n=59)$; and myocardial infarction, $9 \%(n=45)$. Adverse event rates were within the $95 \%$ confidence interval (CI) of the calculated expected rates (27-35\% for mortality and $39-48 \%$ for morbidity or mortality) [11].

Chuansumrit et al. studied the use of rFVIIa for control and prevention of hemorrhage in nonhemophilic pediatric patients and noted an association of adverse events with the total number of doses patients received. Three of 21 patients (14.2\%) that received at least five doses of rFVIIa had thromboembolic events whereas none of the patients that received four doses or fewer had any adverse events. The three patients that experienced adverse events were 3 of 102 (2.9\%) 
patients involved in cardiac surgery cases. All three had experienced massive hemorrhages with shock despite adequate transfusions of blood products. One patient received a total of seven doses of rFVIIa for a total dose of $280 \mathrm{mcg} / \mathrm{kg}$ at 24 hours post operation. The second patient received a total dose of $520 \mathrm{mcg} / \mathrm{kg}$ divided into 10 doses (within 24 hours of the operation) and the third patient received $840 \mathrm{mcg} / \mathrm{kg}$ divided into nine doses (within 12 hours of the operation). Bleeding was eventually controlled in all patients; however, the first patient experienced multiple cerebral infarctions with associated seizures at 60 hours post final rFVIIa dose. The second patient experienced a cerebral infarction at 24 hours post final rFVIIa dose and the third patient experienced a massive clotted hemothorax with cardiorespiratory compromise at 3 hours post final dose of rFVIIa [31].

Post-operative bleeding is typically managed with administration of blood products and may include: platelets, plasma, cryoprecipitate, and/or red blood cells. Selection of an agent may vary among institutions and patient specific factors [9]. At this time rFVIIa may be considered more of an experimental adjunctive pharmacologic agent, because of a lack of knowledge regarding dosing, safety and efficacy. Our institution has successfully utilized rFVIIa in cardiac surgery patients to control bleeding after exhausting conventional means. We use a standard dose of $1 \mathrm{mg}$ intravenously that may be repeated if necessary after 3 hours.

\section{Cardiac Surgery and Alternate Hemostatic Agents}

Historically Aprotinin, an antifibrinolytic drug, has been used in cardiac surgery to reduce blood loss and need for transfusions; however, it was taken off the market due to increased risk of death and is currently limited to investigational use only [26]. Lysine analogs such as aminocaproic acid or tranexamic acid have been used as a prophylactic measure to reduce bleeding and transfusion requirements [9]. Aminocaproic acid may be given to prevent perioperative bleeding as 10g intravenously (IV) over 20-30 minutes prior to skin incision, followed by $1-2.5 \mathrm{~g} /$ hour until the end of the procedure or for up to 4 hours after protamine reversal. Alternatively, it may be given as $10 \mathrm{~g}$ IV over 20-30 minutes prior to skin incision, followed by $10 \mathrm{~g}$ after heparin is given and then a final $10 \mathrm{~g}$ at the discontinuation of $\mathrm{CPB}$, before heparin reversal [27]. Tranexamic acid is at least seven times more potent than aminocaproic acid and it has a longer half-life [9]. Dosing of tranexamic acid is not yet well established for cardiac surgery. Dowd et al. demonstrated that tranexamic acid is rapidly eliminated in patients undergoing $\mathrm{CPB}$ and suggested two different dosing schemes based on the bleeding risks of the patient. For low-risk patients, Dowd et al. recommends a loading dose of $12.5 / \mathrm{mg} / \mathrm{kg}$ (or greater) given over 30 minute, a maintenance infusion of $6.5 \mathrm{mg} / \mathrm{kg} / \mathrm{hour}$ (or greater) and a CPB priming dose if $1 \mathrm{mg} / \mathrm{kg}$ (or greater). For high-risk patients a loading dose of $30 \mathrm{mg} / \mathrm{kg}$, plus an additional $2 \mathrm{mg} / \mathrm{kg}$ to the pump prime, and a maintenance infusion of $16 \mathrm{mg} / \mathrm{kg} / \mathrm{hour}$ [28]. 
Desmopressin is another agent that has been studied for hemostatic properties in surgery [29, 30]. Crescenzi et al. performed a meta-analysis pooling data from 38 randomized clinical trials and concluded that desmopressin was associated with reduced transfusion requirements (standardized mean difference $=-0.29[-0.52$ to -0.06$]$ units per patient; $\mathrm{P}=0.01$ ). In most of the studies desmopressin was given prophylactically at $0.3 \mathrm{mcg} / \mathrm{kg}$ and was given over 15 to 30 minutes. The risk of thromboembolic events was not statistically significant (5.7\% [57/1002] in the desmopressin group vs $4.6 \%$ [45/979] in the control group; $\mathrm{P}=0.3$ ) [30].

\section{Current and Future Developments}

At this time we are left with a conundrum. Specifically, no guidelines or dosing parameters have been established. Furthermore, such studies may never be performed on a large enough scale, given the diversity and comorbidities of the CPB population, to ever completely elucidate the answer to this question. Until these guidelines are established or these studies performed, the evidence to support the safe use of rFVIIa will be very limited. However, the use of this novel treatment modality should not be completely avoided but should be tailored to meet the clinical needs of each patient by the cardiac surgical team.

\section{Conflict of Interest}

At the time this review was written, the authors did not have any conflicts of interest and were unaware of any conflicts of interest involving the patent authors or authors of any references that were used in the writing of this review.

\section{Acknowledgements}

No external source(s) of funding for the study, for each author or for the manuscript preparation. 


\section{References:}

[1] O'Connell K, Wood J, Wise R, et al. Thromboembolic adverse events after use of recombinant human coagulation factor VIIa. JAMA 2006;295:293-298.

[2] Veldman A, Hoffman M, Ehrenforth S. New insights into the coagulation system and implications for new therapeutic options with recombinant factor VIIa. CurrMed Chem 2003;10:797-811.

[3] Al-Ruzzeh S, Navia JL. The “off-label” role of recombinant factor VIIa in surgery: is the problem deficient evidence or defective concept? J Am Coll Surg 2009;209:659-667

[4] Axelsen, Mads, et al.: US20090018082 (2009).

[5] Skamira C, Stassen JM, Heusel G, Wienen W. Use of activated factor vii for treating thrombolytic therapy-induced major bleedings. US2008028625A1 (2008).

[6] Hedner UKE: US005180583A (1993).

[7] Bevan D. Cardiac bypass haemostasis: putting blood through the mill. Br J Haematol 1999;104:208-219.

[8] Erlich J, Boyle E, Labriola J, et al. Inhibition of the tissue factorthrombin pathway limits infarct size after myocardial ischemia reperfusion injury by reducing inflammation. Am J Pathol 2000;157:1849-1862.

[9] Whitlock R, Crowther MA, Ng Hj. Bleeding in cardiac surgery: its prevention and treatment—an evidence-based review. Crit Care Clin. 2005;21:589-610

[10] Gill R, Herbertson M, et al. Safety and efficacy of recombinant activated factor VII a randomized placebo-controlled trial in the setting of bleeding after cardiac surgery. Circulation. 2009;120:21-27.

[11] Karkouti K, Beattie S, Arellano R, Aye T, Bussieres JS, Callum JL, et al. Comprehensive Canadian review of the off-label use of recombinant activated factor VII in cardiac surgery. Circulation. 2008;118:331-338.

[12] Tatoulis J, Theodore S, et al. Safe use of recombinant activated factor VII for recalcitrant postoperative hemorrhage in cardiac surgery. Interactive Cardiovascular and Thoracic Surgery. 2009;9:459-462.

[13] Romagnoli S, Bevilacqua S, et al. Small-dose recombinant activated factor VII (novoseven) in cardiac surgery. Anesth Analg 2006;102:1320-6. 
[14] Despotis G, Avidan M, Eby C. Prediction and management of bleeding in cardiac surgery. J Throm Haemost 2009; 7 (Suppl. 1): 111-7.

[15] Moulton MJ, Creswell LL, Mackey ME, Cox JL, Rosenbloom M. Reexploration for bleeding is a risk factor for adverse outcomes after cardiac operations. J Thorac Cardiovasc Surg 1996; 111: 1037-46.

[16] Despotis G, Eby C, Lublin DM. A review of transfusion risks and optimal management of perioperative bleeding with cardiac surgery. Transfusion 2008; 48: 2S-30S.

[17] Despotis GJ, Santoro SA, Spitznagel E, Kater KM, Cox JL, Barnes P et al. Prospective evaluation and clinical utility of on-site monitoring of coagulation in patients undergoing cardiac operation. J Thorac Cardiovasc Surg 1994; 107: 271-9.

[18] NIH. Consensus conference. Fresh-frozen plasma. Indications and risks. JAMA 1985; 253: 551-3.

[19] Stehling LC, Donerty DC, Faust RJ, Gerson A, Harrison CR, Landers DF, Laros RK et al. Practice Guidelines for blood component therapy: a report by the American Society of Anesthesiologists Task Force on Blood Component Therapy. Anesthesiology 1996; 84:732-47.

[20] Sørensen B, Asvaldsdottir HS, Gudmundsdottir BR, Onundarson PT. The combination of recombinant factor VIIa and fibrinogen correct clotting ex vivo in patient samples obtained following cardiopulmonary bypass surgery. Thromb Res. 2009 Dec;124(6):695-700. Epub 2009 Sep 4.

[21] Masud F, Bostan F, Chi E, Pass SE, Samir H, Stuebing K, Liebl MG: Recombinant factor VIIa treatment of severe bleeding in cardiac surgery patients: a retrospective analysis of dosing, efficacy, and safety outcomes. J Cardiothorac Vasc Anesth. 2009 Feb;23(1):28-33. Epub 2008 Oct 22.

[22] Willis C, Bird R, Mullany D, Cameron P, Phillips L: Use of rFVIIa for critical bleeding in cardiac surgery: dose variation and patient outcomes. Vox Sang. 2010 May;98(4):531-7. Epub 2009 Oct 29.

[23] Chapman AJ, Blount AL, Davis AT, Hooker RL: Recombinant factor VIIa (NovoSeven RT) use in high risk cardiac surgery. Eur J Cardiothorac Surg. 2011 May 19. [Epub ahead of print]

[24] Zangrillo A, Mizzi A, Biondi-Zoccai G, Bignami E, Calabrò MG, Pappalardo F et al. Recombinant activated factor VII in cardiac surgery: a meta-analysis. J Cardiothorac Vasc Anesth. 2009 Feb;23(1):34-40. Epub 2008 Dec 10. 
[25] Ponschab M, Landoni G, Biondi-Zoccai G, Bignami E, Frati E, Nicolotti D, et al. Recombinant Activated Factor VII Increases Stroke in Cardiac Surgery: A Meta-analysis. J Cardiothorac Vasc Anesth. 2011 May 17. [Epub ahead of print]

[26] Manufacturer removes remaining stocks of trasylol access limited to investigational use. [internet] FDA News Release. 2008 May 14. Available from: http://www.fda.gov/NewsEvents/Newsroom/PressAnnouncements/2008/ucm116895.htm. (accessed on: June 30, 2011)

[27] Aminocaproic acid [internet]. Uptodate; 2011 [cited 2011 Jun 30]. Available from: www.uptodate.com. (accessed on: June 30, 2011)

[28] Dowd NP, Karski JM, Cheng DC, Carroll JA, Lin Y, James RL, Butterworth J: Pharmacokinetics of tranexamic acid during cardiopulmonary bypass. Anesthesiology. 2002; 97:390-9

[29] Drummond JC, Petrovitch CT, Lane TA. Clinical anesthesia. 6th ed. Philadelphia : Lippincott Williams \& Wilkins; c2009. Chapter 16, Hemostasis and transfusion medicine; p. 404-405

[30] Crescenzi G, Landoni G, Biondi-Zoccai G, Pappalardo F, Nuzzi M, Bignami E, et al. Desmopressin reduces transfusion needs after surgery. Anesthesiology. 2008; 109:1063-76

[31] Chuansumrit A, Teeraratkul S, Wanichkul S, Treepongkaruna S, Sirachainan N, Pakakasama S, et al. Recombinant-activated factor vii for control and prevention of hemorrhage in nonhemophilic pediatric patients. Blood Coagulation and Fibrinolysis 2010,21:354-362 